EUROPEAN JOURNAL OF PURE AND APPLIED MATHEMATICS

Vol. 15, No. 1, 2022, 64-81

ISSN 1307-5543 - ejpam.com

Published by New York Business Global

\title{
On the Independent Neighborhood Polynomial of the Rooted Product of Two Trees
}

\author{
Normalah S. Abdulcarim ${ }^{1, *}$, Susan C. Dagondon ${ }^{2}$ \\ 1 Department of Mathematics, College of Natural Sciences and Mathematics, \\ Mindanao State University Main Campus, 9700 Marawi City, Philippines \\ 2 Department of Mathematics and Statistics, College of Science and Mathematics, \\ Center of Graph Theory, Algebra, and Analysis-Premier Research Institute of Science and \\ Mathematics, Mindanao State University-Iligan Institute of Technology, 9200 Iligan City, \\ Philippines
}

\begin{abstract}
Let $G$ be a connected graph. We say that a given graph is a tree if every pair of vertices is connected by a unique path. The rooted product of two trees is relevant to tree, as the obtained product is another tree. In this paper, we establish the independent neighborhood sets of a tree and obtain its corresponding independent neighborhood polynomial. Furthermore, the independent neighborhood polynomial of the rooted product of two trees were determine using their independent neighborhood sets.
\end{abstract}

2020 Mathematics Subject Classifications: 05C05, 05C76, 05C31

Key Words and Phrases: Rooted Graph, Rooted Product of Graphs, Independent Neighborhood Polynomial

\section{Introduction}

A graph $G$ is a pair $(V(G), E(G))$ consisting of a nonempty finite set of vertices $V(G)$ and a set of edges $E(G)$ of unordered pairs of elements of $V(G)$. The cardinalities of $V(G)$ and $E(G)$ are called the order and size of $G$, respectively. We write $x=u v$ and say that $u$ and $v$ are adjacent vertices; vertex $u$ and edge $x$ are incident with each other, so are $v$ and $x$. The two vertices incident with an edge are its end vertices or ends, and an edge joins its ends. Two vertices of a graph $G$ are said to be neighbors if they are adjacent in $G$.

The neighborhood of a vertex $v \in V(G)$ is the set $N(v)=\{w: w \in V(G)$ and $v w \in$ $E(G)\}$. A vertex $v$ is pendant if its neighborhood contains only one vertex; and edge $e=u v$ is pendant if one of its end vertices is a pendant vertex.

*Corresponding author.

DOI: https://doi.org/10.29020/nybg.ejpam.v15i1.4220

Email addresses: normalah.abdulcarim@msumain.edu.ph (N. S. Abdulcarim), susan.dagondon@g.msuiit.edu.ph (S. C. Dagondon) 
A path is a nonempty graph $P=(V(P), E(P))$ of the form

$$
V(P)=\left\{v_{1}, \cdots, v_{m}\right\}, \quad E(P)=\left\{v_{1} v_{2}, v_{2} v_{3}, \cdots, v_{m-1} v_{m}\right\},
$$

where the $v_{i}$ are all distinct. If $E=\left\{v_{1} v_{2}, v_{2} v_{3}, \cdots, v_{m-1} v_{m}, v_{m} v_{1}\right\}$, then the graph is called a cycle.

In this study, we consider the graph polynomial. Some familiar examples include chromatic polynomial by George David Birkhoff [1], matching polynomial by Farrel [4], independence polynomial by Gutman and Harary [7], and by Levit and Mandrescu [10]. Recently, another type of graph polynomial, the neighborhood polynomial were studied by some researchers. Some of these are the articles "Neighborhood Polynomial of Graphs" by J. Brown and R. Nowakowski [9] and "On the Independent Neighborhood Polynomial of Graphs" by K.B. Murthy and Puttaswamy[11]. The main interest of this study is to establish results on the independent neighborhood polynomial of tree and the rooted product of two trees.

\section{Preliminaries}

This section presents some basic concepts in graph theory and known results needed in this study.

Definition 1. [2] The open neighborhood of a vertex $x$, denoted by $N(x)$, is a set containing all vertices $y$ which are adjacent to $x$, that is, $N(x)=\{y \in V(G): x y \in E(G)\}$. In case $N(x)$ is a singleton, $x$ is an end-vertex. The closed neighborhood of a vertex $x$ of $G$ is the set $N[x]=N(x) \cup\{x\}$.

Definition 2. [11] A set $S$ of vertices in a graph $G$ is a neighborhood set if $G=\bigcup_{u \in S}\langle N[u]\rangle$ where $\langle N[u]\rangle$ is the subgraph of $G$ induced by $u$ and all the vertices adjacent to $u$. The neighborhood number of $G$ is the minimum cardinality of neighborhood sets, denoted by $\eta_{i}(G)$.

Example 1. Consider the graph $H$ in Figure 1.

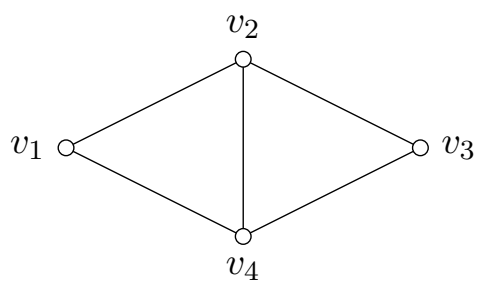

Figure 1: A graph $H$ 
The neighborhood sets of $H$ are $\left\{v_{1}, v_{3}\right\},\left\{v_{2}\right\},\left\{v_{4}\right\},\left\{v_{1}, v_{2}\right\},\left\{v_{2}, v_{3}\right\},\left\{v_{2}, v_{4}\right\}\left\{v_{1}, v_{4}\right\}$, $\left\{v_{3}, v_{4}\right\},\left\{v_{1}, v_{2}, v_{3}\right\},\left\{v_{1}, v_{2}, v_{4}\right\},\left\{v_{1}, v_{3}, v_{4}\right\},\left\{v_{2}, v_{3}, v_{4}\right\}$ and $\left\{v_{1}, v_{2}, v_{3}, v_{4}\right\}$. Here,

$$
\eta_{i}(H)=1 .
$$

Definition 3. [11] A set $S \subseteq V(G)$ is an independent neighborhood set of $G$, if $S$ is a neighborhood set and no two vertices in $S$ are adjacent.

Definition 4. [11] Let $G=(V(G), E(G))$ be a graph with $m$ vertices. Then the independent neighborhood polynomial of $G$ of order $m$ is

$$
N_{i}(G, x)=\sum_{j=\eta_{i}(G)}^{m} n_{i}(G, j) x^{j},
$$

where $n_{i}(G, j)$ is the number of independent neighborhood set of $G$ of size $j$ and $\eta_{i}(G)$ is the minimum cardinality of an independent neighborhood set which is called the independent neighborhood number of $G$.

Example 2. In Figure 1 above, the only independent neighborhood sets of $H$ are $\left\{v_{2}\right\},\left\{v_{4}\right\}$ and $\left\{v_{1}, v_{3}\right\}$. Therefore, the independent neighborhood polynomial of $H$ is

$$
N_{i}(H, x)=2 x+x^{2} .
$$

Definition 5. [6]A graph is acyclic if it has no cycles. A graph is said to be connected if every pair of vertices are joined by a path. A graph that is not connected is called disconnected. A tree is a connected acyclic graph.

Theorem 1. [8] For a simple graph $G$ (with $n$ vertices, $n \geq 1$ ), the following statements are equivalent

(i) $G$ is a tree

(ii) $G$ is connected and has no cycles

(iii) $G$ is connected and has $n-1$ edges

(iv) $G$ has $n-1$ edges and has no cycles

(v) Every two vertices of $G$ are joined by a unique path

Corollary 1. [8] Every nontrivial tree has at least two end vertices.

Definition 6. [3] Let $G$ be a graph. The distance between two vertices $x$ and $y$ in a graph $G$, denoted by $d_{G}(x, y)$ or simply $d(x, y)$, is the length of the shortest path joining them, otherwise, $d(x, y)=\infty$.

Remark 1. [6] In a connected graph $G$, the distance is a metric, that is, for all $x, y, z \in V(G)$, 
(i) $d(x, y) \geq 0$, with $d(x, y)=0$ if and only if $x=y$.

(ii) $d(x, y)=d(y, x)$

(iii) $d(x, y)+d(y, z) \geq d(x, z)$.

Remark 2. For any tree $T, d(x, y)+d(y, z)=d(x, z)$.

Example 3. Consider the tree below

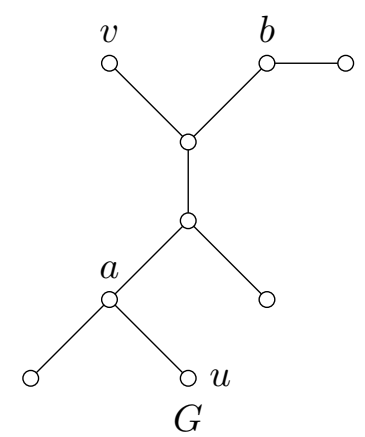

Figure 2: A tree

In Figure 2, the distance between vertices $u$ and $v$ is $d(u, v)=4$ and the distance between $a$ and $b$ is $d(a, b)=3$.

Definition 7. [6] A graph in which one vertex is fixed as a root vertex to distinguish it from the other is called a rooted graph. The rooted product of a graph $G$ and a rooted graph $H$ is defined as follows:

Let $V(G)=\left\{g_{1}, g_{2}, \cdots, g_{n}\right\}, V(H)=\left\{h_{1}, h_{2}, \cdots, h_{m}\right\}$ and that root vertex of $H$ is $h_{1}$, we define $G \bullet H=(V, E)$ where $V=\left\{\left(g_{i}, h_{j}\right): 1 \leq i \leq n, 1 \leq j \leq m\right\}$ and $E=\left\{\left(g_{i}, h_{1}\right)\left(g_{k}, h_{1}\right): g_{i} g_{k} \in E(G)\right\} \cup \bigcup_{i=1}^{n}\left\{\left(g_{i}, h_{j}\right)\left(g_{i}, h_{k}\right): h_{j} h_{k} \in E(H)\right\}$.

Example 4. Let $G$ be a graph and $H$ be a rooted graph with $h_{1}$ as its root vertex. Then the rooted product of $G$ and $H$ is shown in Figure 3. 


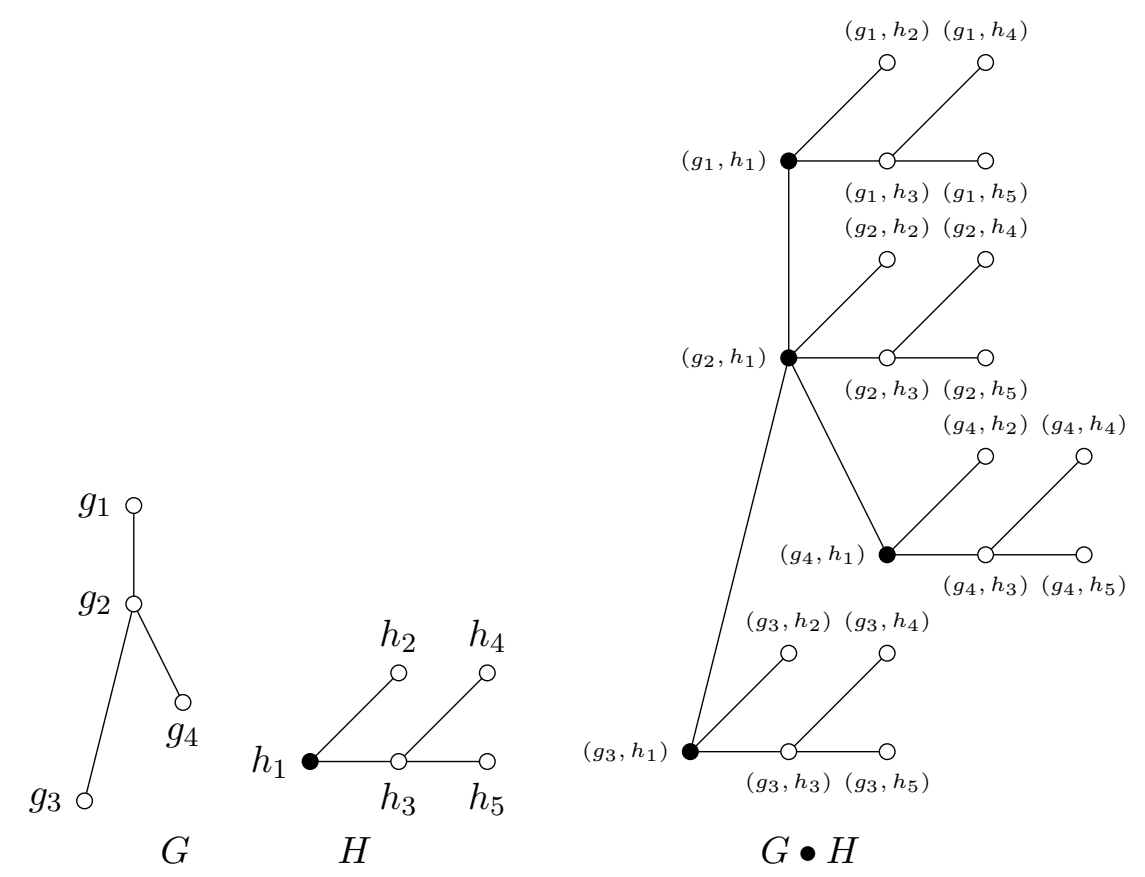

Figure 3: The rooted product $G \bullet H$ of graph $G$ and rooted graph $H$

Definition 8. [5] Let $S$ and $T$ be sets. A function $f: S \longrightarrow T$ is one-to-one (injective) if for all $s_{1}, s_{2} \in S, f\left(s_{1}\right)=f\left(s_{2}\right) \Longleftrightarrow s_{1}=s_{2}$.

Definition 9. [5] Let $S$ and $T$ be sets. A function $f: S \longrightarrow T$ is onto (surjective) if for all $t \in T$, there exists $s \in S$ such that $f(s)=t$.

Definition 10. [5] Let $S$ and $T$ be sets. A function $f: S \longrightarrow T$ is bijective if $f$ is both injective and surjective.

Definition 11. [5] Graphs $G$ and $H$ are isomorphic, denoted by $G \cong H$, if there exists a bijective mapping $f: V(G) \longrightarrow V(H)$ such that $u v \in E(G)$ if and only if

$$
f(u) f(v) \in E(H) .
$$

\section{Independent Neighborhood Polynomial Trees}

In this section, we generalize the independent neighborhood set of any tree and represent it in an independent neighborhood polynomial of a graph.

Proposition 1. Let $G$ be a connected graph, and $S \subseteq V(G)$. Then $S$ is an independent neighborhood set of $G$ if and only if the following hold:

(i) $S$ is an independent set

(ii) For each $u v \in E(G)$, there exists $w \in S$ such that $u, v \in N[w]$. 
Proof. Assume that $S$ is an independent neighborhood set of $G$. Then $S$ is an independent set and $(i)$ holds. Let $u v \in E(G)$. Since $G=\bigcup_{w \in S}\langle N[w]\rangle$, there exists $w \in S$ for with $u v \in E(\langle N[w]\rangle)$. This means that $u, v \in N[w]$, and (ii) holds.

Suppose that $(i)$ and $(i i)$ hold for $S$. Let $v \in V(G)$ and let $u \in V(S) \backslash\{v\}$ for which $u v \in E(G)$. By $(i i)$, there exists $w \in S$ such that $u, v \in N[w]$. Thus, $v \in V(\langle N[w]\rangle)$. Since $v$ is arbitrary, $V(G)=V\left(\bigcup_{w \in S}\langle N[w]\rangle\right)$. Let $u v \in E(G)$. By (ii) again, there exists $w \in S$ for which $u, v \in N[w]$. Hence, $u v \in E(\langle N[w]\rangle)$. Thus, $u v \in E\left(\bigcup_{w \in S}\langle N[w]\rangle\right)$. Hence, $E(G)=E\left(\bigcup_{w \in S}\langle N[w]\rangle\right)$. Therefore, $S$ is an independent neighborhood set of $G$.

Proposition 2. Let $G$ be any IN-graph. If $\Omega$ is an independent neighborhood set of $G$, then there is no proper subset of $\Omega$, say $\Delta$, such that $\bigcup_{v \in \Delta}\langle N[v]\rangle=G$.

Proof. Let $G$ be any $I N$-graph. Let $\Omega$ be an independent neighborhood set of $G$. Assume to the contrary that there exists a proper subset $\Delta$ of $\Omega$ such that $\bigcup_{v \in \Delta}\langle N[v]\rangle=G$. Let $u \in \Omega \backslash \Delta$. Since $\Omega$ is an independent neighborhood set, every vertices in $\Omega$ are not adjacent. This implies $u \in \Omega \backslash \Delta$ is not a neighborhood of $\Delta$. It follows that $u$ is not in $\bigcup_{v \in \Delta}\langle N[v]\rangle$. This is a contradiction for $\bigcup_{v \in \Delta}\langle N[v]\rangle=G$. Hence, there is no proper subset of $\Omega$ that is also an independent neighborhood set of $G$.

Corollary 2. Let $G$ be an IN-graph. If $\Omega$ is an independent neighborhood set of $G$, then there is no independent neighborhood set of $G$ that contains $\Omega$.

Proof. Let $\Omega$ be an independent neighborhood set of an $I N$-graph $G$. Assume that there exists an independent neighborhood set of $G$ that contains $\Omega$, say $\Delta$. Let $\Delta=\Omega \cup\left\{x_{1}, x_{2}, \cdots, x_{n}\right\}$ for some $x_{i} \in V(G)$. Since $\Delta$ is an independent neighborhood set of $G$, for all $v_{j} \in \Omega$ and $x_{i}, v_{j^{\prime} s}$ and $x_{i^{\prime} s}$ are non adjacent. This implies $x_{i} \notin N\left[v_{j}\right]$ for all $v_{j} \in \Omega, i=1, \cdots, n$. It follows that $x_{i}$ is not in $\bigcup_{v \in \Omega}\langle N[v]\rangle$. Hence, $\bigcup_{v \in \Omega}\langle N[v]\rangle \neq G$. This is a contradiction to our assumption that $\Omega$ is an independent neighborhood set of $G$. Therefore, there is no independent neighborhood set of $G$ that contains $\Omega$.

For the succeeding results, we let $\mathbb{N}^{*}=\mathbb{N} \cup\{0\}$. 
Theorem 2. Let $G$ be any tree. Then for any $u \in V(G)$, the set

$$
\Omega=\left\{v \in V(G): d(u, v)=2 n, n \in \mathbb{N}^{*}\right\}
$$

is an independent neighborhood set of $G$.

Proof. Let $u \in V(G)$. Let $\Omega=\left\{v \in V(G): d(u, v)=2 n, n \in \mathbb{N}^{*}\right\}$. Clearly, for any $v_{1}, v_{2} \in \Omega, v_{1}$ and $v_{2}$ are not adjacent. We are left to show that $\bigcup_{\omega \in \Omega}\langle N[\omega]\rangle=G$. Assume to the contrary that $\bigcup_{\omega \in \Omega}\langle N[\omega]\rangle \neq G$. Then there exists $x y \in E(G)$ such that $x y \notin E\left(\bigcup_{\omega \in \Omega}\langle N[\omega]\rangle\right)$. It follows that both $x, y \notin \Omega$. Observe that for any $\omega \in \Omega, d(\omega, x)=$ $2 m+1, m \in \mathbb{N}^{*}$. Now,

$$
\begin{aligned}
d_{G}(\omega, y) & =d_{G}(\omega, x)+d_{G}(x, y) \\
& =(2 m+1)+1 \\
& =2 m+2 \\
& =2(m+1) .
\end{aligned}
$$

Let $n=m+1$. Then $n \in \mathbb{N}^{*}$ and $d(\omega, y)=2 n$ which implies $y \in \Omega$, a contradiction since $y \notin \Omega$. Hence, $\bigcup_{\omega \in \Omega}\langle N[\omega]\rangle=G$. Therefore, $\Omega$ is an independent neighborhood set of $G$.

Corollary 3. Let $F$ be the set of nonpendant vertices in a tree $G$ and $f \in F$. Let

$$
A=\left\{a \in F: d_{G}(a, f)=2 n, n \in \mathbb{N}^{*}\right\}
$$

and

$$
B=F \backslash A \text {. }
$$

Then the sets

$$
\Omega=\{v: v \text { is a pendant neighbor of } a \text {, for some } a \in A\} \cup B
$$

and

$$
\Delta=\{u: u \text { is a pendant neighbor of } b, \text { for some } b \in B\} \cup A
$$

are the only independent neighborhood sets of $G$.

Proof. Let $F$ be the set of nonpendant vertices in $G$ and $f \in F$. Let

$$
A=\left\{a \in F: d_{G}(a, f)=2 n, n \in \mathbb{N}^{*}\right\}
$$

and

$$
B=F \backslash A \text {. }
$$


Then for every $a \in A$ and $b \in B, d_{G}(a, b)=2 m+1, m \in \mathbb{N}^{*}$. We note that for any $u \in N(g), d_{G}(u, g)=1$ for every $g \in G$. Let $v$ be a pendant neighbor of $a \in A$. Now, for any $b \in B$, we have

$$
\begin{aligned}
d_{G}(v, b) & =d_{G}(v, a)+d_{G}(a, b) \\
& =1+2 m+1 \\
& =2(m+1) .
\end{aligned}
$$

Let $n=m+1$. Then $n \in \mathbb{N}^{*}$ and $d_{G}(v, b)=2 n$ which is even. By Theorem $2, \Omega$ is an independent neighborhood set of $G$. Similarly, we can show that $\Delta$ is also an independent neighborhood set of $G$.

We are left to show that $\Omega$ and $\Delta$ are the only independent neighborhood sets of $G$. Clearly, from the definition of $\Omega$ and $\Delta, \Omega^{c}=\Delta$. Assume that there is another independent neighborhood set of $G$, say $\Lambda$. Define $\Lambda=\Omega_{1} \cup \Delta_{1}$ where $\Omega_{1} \subset \Omega$ and $\Delta_{1} \subset \Delta$. Let $x \in \Omega \backslash \Omega_{1}$ and $y \in \Delta \backslash \Delta_{1}$. Suppose that $x w, y z \in E(G)$ for some $w, z \in \Lambda$. Since $\Omega$ and $\Delta$ are independent neighborhood sets of $G, w$ must be in $\Delta_{1}$ and $z$ must be in $\Omega_{1}$. Observe that $d_{G}(w, z)=2 r$ for some $r \in \mathbb{N}^{*}$ and $d_{G}(y, z)=1$ since $\Lambda$ is an independent neighborhood set of $G$ and $y z \in E(G)$. Now,

$$
\begin{aligned}
d_{G}(w, y) & =d_{G}(w, z)+d_{G}(y, z) \\
& =2 n+1 .
\end{aligned}
$$

This means $d_{G}(w, y)$ is odd which is a contradiction since $\Delta$ is an independent neighborhood set of $G$. Hence, $\Lambda$ cannot be an independent neighborhood set of $G$. Therefore, $S$ and $T$ are the only independent neighborhood set of $G$.

Corollary 4. Let $F$ be the set of nonpendant vertices in a tree $G$ and $f \in F$. Let

$$
A=\left\{a \in F: d_{\langle F\rangle}(a, f)=2 n, n \in \mathbb{N}^{*}\right\}
$$

and

$$
B=F \backslash A \text {. }
$$

Suppose that

$$
\Omega=\{v: v \text { is a pendant neighbor of } a \in A\} \cup B
$$

and

$$
\Delta=\{u: u \text { is a pendant neighbor of } b \in A\} \cup A .
$$

Then

$$
N_{i}(G, x)=x^{|\Omega|}+x^{|\Delta|} .
$$

Example 5. Consider $u=5 \in V(G)$ in the graph in Figure 4. 


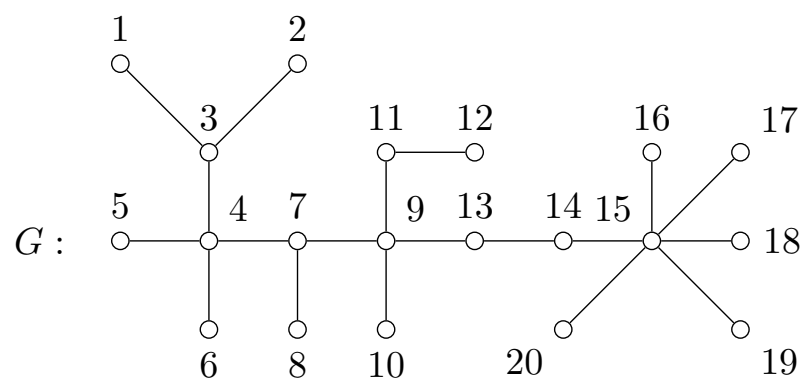

Figure 4: A tree $G$

Observe that for every $u$ in the set $\Omega=\{3,5,6,7,10,11,13,15\}, d_{G}(u, 5)=2 n, n \in \mathbb{N}^{*}$. By Theorem 2, $\Omega$ is an independent neighborhood set of $G$. Also, if we consider $u=1 \in$ $V(G)$, for each $v$ in the set $\Delta=\{1,2,4,8,9,12,14,16,17,18,19,20\}, d_{G}(v, 1)=2 m, m \in$ $\mathbb{N}^{*}$. By Theorem 3.2.4, $\Delta$ is also an independent neighborhood set of $G$. We note that $|\Omega|=8$ and $|\Delta|=12$. Therefore,

$$
N_{i}(G, x)=x^{8}+x^{12} .
$$

Other Solution: From Figure 4, we can see that the set of nonpendant vertices in $G$ is given by $F=\{3,4,7,9,11,13,14,15\}$. Consider $f=3 \in F$. Then $A=\{3,7,11,13,15\}$ and $B=F \backslash A=\{4,9,14\}$. Applying Corollary 3, the independent neighborhood sets of $G$ are

$$
\begin{aligned}
\Omega & =\{v: v \text { is a pendant neighbor of } a, \text { for some } a \in A\} \cup B \\
& =\{1,2,8,12,16,17,18,19,20\} \cup\{4,9,14\} \\
& =\{1,2,4,8,9,12,14,16,17,18,19,20\}
\end{aligned}
$$

and

$$
\begin{aligned}
\Delta & =\{v: v \text { is a pendant neighbor of } b, \text { for some } b \in B\} \cup A \\
& =\{5,6,10\} \cup\{3,7,11,13,15\} \\
& =\{3,5,6,7,10,11,13,15\} .
\end{aligned}
$$

Therefore, by Corollary 4,

$$
N_{i}(G, x)=x^{8}+x^{12} .
$$

Corollary 5. For any tree $T$, if $\Omega$ and $\Delta$ are the independent neighborhood sets of $T$, then

$$
\Omega \cup \Delta=V(T) .
$$

Proof. Let $\Omega$ and $\Delta$ be the independent neighborhood sets of a tree $T$. Assume to the contrary that $\Omega \cup \Delta \neq V(T)$. Then there exists $v \in V(T)$ such that $v \notin \Omega \cup \Delta$. This implies $v \notin \Omega$ and $v \notin \Delta$. Since $\Omega$ and $\Delta$ are both independent neighborhood sets of $T$, there 
exists $g \in \Omega$ and $h \in \Delta$ such that $v \in N[g]$ and $v \in N[h]$. This shows that $d(v, g)=1$ and $d(v, h)=1$ and follows that $d(g, h)=d(v, g)+d(v, h)=2$. Thus, $\Omega \cup\{h\}$ and $\Delta \cup\{g\}$ are independent neighborhood sets of $T$ which is a contradiction by Corollary 3 . Hence, $\Omega \cup \Delta=V(T)$.

Corollary 6. For any tree $T$, if $\Omega$ and $\Delta$ are the independent neighborhood sets of $T$, then

$$
\Omega \cap \Delta=\varnothing .
$$

Proof. Let $\Omega$ and $\Delta$ be the independent neighborhood sets of a tree $T$. Assume to the contrary that $\Omega \cap \Delta \neq \varnothing$. This implies there exists $u \in \Omega \cap \Delta$. It follows that $u \in \Omega$ and $u \in \Delta$. Since $\Omega$ and $\Delta$ are both independent neighborhood sets of $T, d(u, g)=2 n, \forall g \in \Omega$ and $d(u, h)=2 m, \forall h \in \Delta$ for some $n, m \in \mathbb{N}^{*}$. Consequently,

$$
d(g, h)=d(g, u)+d(u, h)=2 n+2 m=2(n+m) .
$$

This shows that $\Omega \cup \Delta$ is an independent neighborhood set of $T$. But $\Omega \cup \Delta=V(T)$ which is clearly not an independent neighborhood set of $T$. Hence, $\Omega \cap \Delta=\varnothing$.

Corollary 7. For any tree $T$ with independent neighborhood sets $\Omega$ and $\Delta$, if uv $\in E(T)$, then $u \in \Omega$ and $v \in \Delta$.

Proof. Let $T$ be a tree with independent neighborhood sets $\Omega$ and $\Delta$. Let $u v \in E(T)$. Suppose that $u \notin \Omega$ or $v \notin \Delta$. Consider the following cases.

case 1: $u \notin \Omega$ and $v \in \Delta$

Since $u v \in E(T)$ and $\Omega$ is an independent neighborhood set of $T$, by Proposition 1 , there exists $w \in \Omega$ such that $u, v \in N[w]$. But the set of edges $\{u w, v w, u v\}$ forms a cycle which is a contradiction since $T$ is a tree.

case 2: $v \notin \Delta$ and $u \in \Omega$

By Proposition 1, being $\Delta$ an independent neighborhood set of $T$ implies there exists $z \in \Delta$ such that $u, v \in N[z]$. Observe that the edges $u z, v z, u v$ form a cycle which is a contradiction since $T$ is acylic.

case 3: $u \notin \Omega$ and $v \notin \Delta$

Since $\Omega$ and $\Delta$ are independent neighborhood sets of $T$, there exist $x \in \Omega$ and $y \in \Delta$ such that $u, v \in N[x]$ and $u, v \in N[y]$ by Proposition 1 . The set of edges $\{u x, v x, u v\}$ and $\{u y, v y, u v\}$ are all cycle which is a contradiction since $T$ is acyclic.

Thus, in either of the cases, we arrived at a contradiction. Therefore, $u \in \Omega$ and $v \in \Delta$.

\section{Independent Neighborhood Polynomial of the Rooted Product of Two Trees}

In this section, we establish the independent neighborhood sets of the rooted product of a tree and a rooted tree using the independent neighborhood sets of each tree and represent it in an independent neighborhood polynomial of a graph. 
Theorem 3. Let $G$ be any tree and $H$ be a rooted tree with root vertex $u$. Then the distance $d((x, y),(w, z))$ between two vertices $(x, y),(w, z)$ of the rooted product graph $G \bullet H$ is given by:

1. $d((x, y),(w, z))=d(y, z)$ if $x=w$,

2. $d((x, y),(w, z))=d(x, w)$ if $y=u=z$,

otherwise,

3. $d((x, y),(w, z))=d((x, y),(x, u))+d((x, u),(w, u))+d((w, u),(w, z))$.

Proof. 1. Let $S=\{(x, y),(w, z) \in V(G \bullet H): x=w\}$. We claim that $\langle S\rangle \cong H$. First, we define the map function $\beta: S \rightarrow V(H)$ given by $(x, y) \mapsto y$ for $y \in H$. For $\left(x, y_{1}\right),\left(x, y_{2}\right) \in S$ such that $\beta\left(\left(x, y_{1}\right)\right)=\beta\left(\left(x, y_{2}\right)\right)$, we have $y_{1}=\beta\left(\left(x, y_{1}\right)\right)=\beta\left(\left(x, y_{2}\right)\right)=$ $y_{2}$. This shows that $\beta$ is one-to-one. Since $S=\{(x, y) \in V(G \bullet H): x \in V(G), y \in V(H)\}$, for every $h \in V(H)$, there exists $g \in V(G)$ such that $(g, h) \in V(G \bullet H)$. Observe that $h=\beta((g, h))$. Hence, $\beta$ is onto. Lastly, we show that $\beta$ preserves adjacency. Let $\left(g, h_{1}\right),\left(g, h_{2}\right) \in S \subseteq V(G \bullet H)$ such that $\left(g, h_{1}\right)\left(g, h_{2}\right) \in E(G \bullet H)$. By Definition 7 , $h_{1} h_{2} \in E(H)$. Thus, $\left(g, h_{1}\right)\left(g, h_{2}\right) \in E(G \bullet H) \Leftrightarrow \beta\left(\left(g, h_{1}\right)\right) \beta\left(\left(g, h_{2}\right)\right)=h_{1} h_{2} \in E(H)$. Hence, $\beta$ preserves adjacency. Therefore, $\langle S\rangle \cong H$. Consequently, $d((x, y),(w, z))=$ $d(y, z)$ for $x=w$.

2. Let $T=\{(x, y),(w, z) \in V(G \bullet H): y=u=z\}$. We claim that $\langle T\rangle \cong G$. First, we define the map function $\gamma: T \rightarrow V(G)$ given by $(x, y) \mapsto x$. Let $\left(x_{1}, u\right),\left(x_{2}, u\right) \in T$. If $\gamma\left(\left(x_{1}, u\right)\right)=\gamma\left(\left(x_{2}, u\right)\right)$, then $x_{1}=\gamma\left(\left(x_{1}, u\right)\right)=\gamma\left(\left(x_{2}, u\right)\right)=x_{2}$. This implies $\gamma$ is oneto-one. Since for every $g_{1} \in V(G), \gamma((g, u))=g, \gamma$ is onto. Lastly, let $\left(g_{1}, u\right),\left(g_{2}, u\right) \in$ $V(G \bullet H)$ such that $\left(g_{1}, u\right)\left(g_{2}, u\right) \in E(G \bullet H)$. By Definition $7, g_{1} g_{2} \in E(G)$. Now, $\left(g_{1}, u\right)\left(g_{2}, u\right) \in E(G \bullet H) \Leftrightarrow \gamma\left(\left(g_{1}, u\right)\right) \gamma\left(\left(g_{2}, u\right)\right)=g_{1} g_{2} \in E(G)$. Hence, $\gamma$ preserves adjacency. Thus, $\langle T\rangle \cong G$. Therefore, $d((x, y),(w, z))=d(x, w)$ for $y=u=z$.

3. Since $G \bullet H$ is a tree, every vertices of $G \bullet H$ is connected by a unique path. By definition of rooted product, for vertices $(x, y),(w, z)$ where $x \neq w$ and $y \neq z$, the vertices $(x, y)$ and $(w, z)$ is connected by the paths $(x, y)-(x, u)$ path, $(x, u)-(w, u)$ path and $(w, u)-(w, z)$ path where $u$ is the root vertex of graph $H$. Hence, by Remark 2 ,

$$
d((x, y),(w, z))=d((x, y),(x, u))+d((x, u),(w, u))+d((w, u),(w, z)) .
$$

Remark 3. Let $G$ be a tree graph, $H$ be a rooted tree and $u$ be its rooted vertex. If $y=u$ and $z \neq u$, then

$$
\begin{aligned}
d((x, y),(w, z)) & =d((x, y),(x, u))+d((x, u),(w, u))+d((w, u),(w, z)) \\
& =0+d((x, u),(w, u))+d((w, u),(w, z)) \\
& =d((x, u),(w, u))+d((w, u),(w, z)) \\
& =d(x, w)+d(u, z) .
\end{aligned}
$$


Theorem 4. Let $G$ and rooted $H$ be trees with independent neighborhood sets $\Omega_{1}, \Delta_{1}$ and $\Omega_{2}, \Delta_{2}$, respectively. Then the independent neighborhood sets of $G \bullet H$ are

$$
\left\{(x, y): x \in \Omega_{1}, y \in \Omega_{2}\right\} \cup\left\{(w, z): w \in \Delta_{1}, z \in \Delta_{2}\right\}
$$

and

$$
\left\{(w, y): w \in \Delta_{1}, y \in \Omega_{2}\right\} \cup\left\{(x, z): x \in \Omega_{1}, z \in \Delta_{2}\right\} .
$$

Proof. Let $G$ and rooted $H$ be trees with independent neighborhood sets $\Omega_{1}, \Delta_{1}$ and $\Omega_{2}, \Delta_{2}$, respectively. Let $u$ be the root vertex of $H$ and that without loss of generality, let $u \in \Omega_{2}$. We will show that the sets

$$
\left\{(x, y): x \in \Omega_{1}, y \in \Omega_{2}\right\} \cup\left\{(w, z): w \in \Delta_{1}, z \in \Delta_{2}\right\}
$$

and

$$
\left\{(w, y): w \in \Delta_{1}, y \in \Omega_{2}\right\} \cup\left\{(x, z): x \in \Omega_{1}, z \in \Delta_{2}\right\}
$$

are the independent neighborhood sets of $G \bullet H$. Now, for any $x \in \Omega_{1}$, $y \in \Omega_{2}, w \in \Delta_{1}$ and $z \in \Delta_{2}$,

$$
d((x, y),(w, z))=d((x, y),(x, u))+d((x, u),(w, u))+d((w, u),(w, z)) .
$$

Since $\Omega_{2}$ is an independent neighborhood set of $H$, by Theorem 2 ,

$$
d((x, y),(x, u))=d(y, u)=2 m, m \in \mathbb{N}^{*} .
$$

Observe that $d((x, u),(w, u))=d(x, w)=2 n+1, n \in \mathbb{N}^{*}$ for $x \in \Omega_{1}, w \in \Delta_{1}$ and $d((w, u),(w, z))=d(u, z)=2 r+1, r \in \mathbb{N}^{*}$ for $u \in \Omega_{2}, z \in \Delta_{2}$. It follows that $d((x, y),(w, z))=2 m+(2 n+1)+(2 r+1)=2(m+n+1)$, which is even and by Theorem 2 , the set

$$
\left\{(x, y): x \in \Omega_{1}, y \in \Omega_{2}\right\} \cup\left\{(w, z): w \in \Delta_{1}, z \in \Delta_{2}\right\}
$$

is an independent neighborhood set of $G \bullet H$. Following same argument for the set

$$
\left\{(w, y): w \in \Delta_{1}, y \in \Omega_{2}\right\} \cup\left\{(x, z): x \in \Omega_{1}, z \in \Delta_{2}\right\}
$$

shows that it is also an independent neighborhood set of $G \bullet H$.

Corollary 8. Let $\Omega_{1}, \Delta_{1}$ and $\Omega_{2}, \Delta_{2}$ be the independent neighborhood sets of trees $G$ and $H$, respectively. Then

$$
N_{i}(G \bullet H, x)=x^{\left|\Omega_{1}\right|\left|\Omega_{2}\right|+\left|\Delta_{1}\right|\left|\Delta_{2}\right|}+x^{\left|\Delta_{1}\right|\left|\Omega_{2}\right|+\left|\Omega_{1}\right|\left|\Delta_{2}\right|} .
$$


Example 6. Consider the graph $G$ and graph $H$ in Figure 5 .
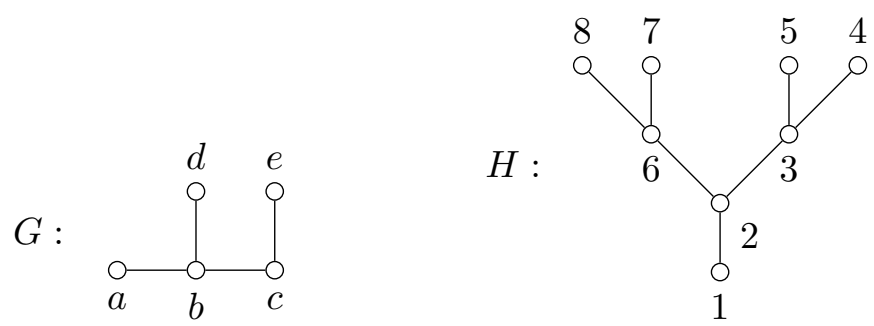

Figure 5: Trees $G$ and $H$

Let the vertex 2 be a rooted vertex and label the rooted tree as $H_{1}$. Then the rooted product of $G$ and $H_{1}$ is given in Figure 6 .

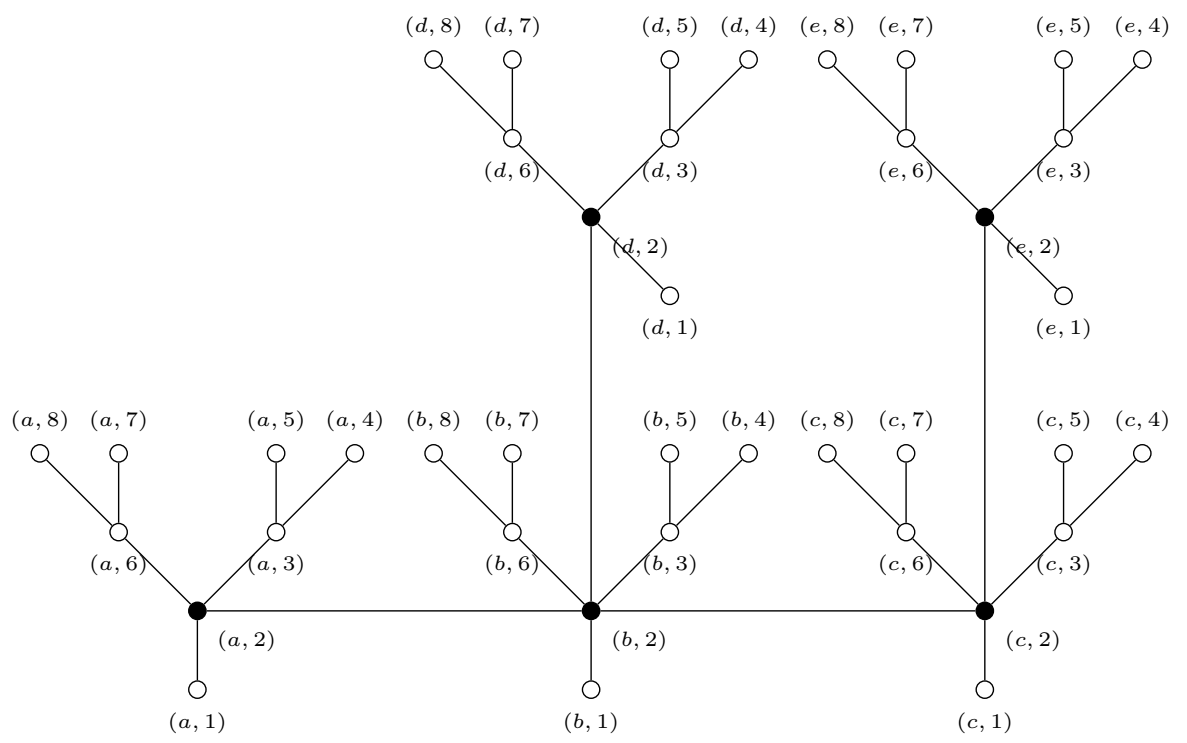

Figure 6: Rooted product of graph $G$ and rooted graph $H_{1}$

We note that the independent neighborhood sets of $G$ are $\Omega_{1}=\{a, d, c\}$,

$\Delta_{1}=\{b, e\}$ and the independent neighborhood sets of $H_{1}$ are $\Omega_{2}=\{2,4,5,7,8\}$, $\Delta_{2}=\{1,3,6\}$. By Theorem 4, the independent neighborhood sets of $G \bullet H_{1}$ are

$$
\begin{aligned}
\left\{(x, y): x \in \Omega_{1}, y \in \Omega_{2}\right\} \cup\left\{(w, z): w \in \Delta_{1}, z \in \Delta_{2}\right\} \\
=\{(a, 2),(a, 4),(a, 5),(a, 7),(a, 8),(d, 2),(d, 4),(d, 5),(d, 7),(d, 8),(c, 2),(c, 4), \\
\quad(c, 5),(c, 7),(c, 8)\} \cup\{(b, 1),(b, 3),(b, 6),(e, 1),(e, 3),(e, 6)\} \\
=\{(a, 2),(a, 4),(a, 5),(a, 7),(a, 8),(d, 2),(d, 4),(d, 5),(d, 7),(d, 8),(c, 2),(c, 4), \\
\quad(c, 5),(c, 7),(c, 8),(b, 1),(b, 3),(b, 6),(e, 1),(e, 3),(e, 6)\}
\end{aligned}
$$


and

$$
\begin{aligned}
\left\{(w, y): w \in \Delta_{1}, y \in \Omega_{2}\right\} \cup\left\{(x, z): x \in \Omega_{1}, z \in \Delta_{2}\right\} \\
=\{(b, 2),(b, 4),(b, 5),(b, 7),(b, 8),(e, 2),(e, 4),(e, 5),(e, 7),(e, 8)\} \cup\{(a, 1),(a, 3), \\
\quad(a, 6),(d, 1),(d, 3),(d, 6),(c, 1),(c, 3),(c, 6)\} \\
=\{(b, 2),(b, 4),(b, 5),(b, 7),(b, 8),(e, 2),(e, 4),(e, 5),(e, 7),(e, 8),(a, 1),(a, 3), \\
\quad(a, 6),(d, 1),(d, 3),(d, 6),(c, 1),(c, 3),(c, 6)\} .
\end{aligned}
$$

Furthermore,

$$
\left|\Omega_{1}\right|=3,\left|\Delta_{1}\right|=2,\left|\Omega_{2}\right|=5 \text { and }\left|\Delta_{2}\right|=3 .
$$

Therefore, by Corollary 8 ,

$$
\begin{aligned}
N_{i}\left(G \bullet H_{1}, x\right) & =x^{3(5)+2(3)}+x^{2(5)+3(3)} \\
& =x^{21}+x^{19} .
\end{aligned}
$$

Theorem 5. Let $G$ be any tree and $H$ be another tree. Suppose $H_{1}$ is a rooted tree from $H$ with rooted vertex $u_{1}$ and $H_{2}$ is also a rooted tree from $H$ with rooted vertex $u_{2}$ where $H_{1} \cong H_{2}$. Then the independent neighborhood polynomial of the rooted product of $G$ and $\mathrm{H}_{2}$ is the same as the independent neighborhood polynomial of the rooted product of $G$ and $H_{1}$, that is,

$$
N_{i}\left(G \bullet H_{2}, x\right)=N_{i}\left(G \bullet H_{1}, x\right) .
$$

Proof. Let $G$ and $H$ be trees with independent neighborhood sets $\Omega_{1}, \Delta_{1}$ and $\Omega_{2}, \Delta_{2}$, respectively. Suppose $H_{1}$ is a rooted tree from $H$ with rooted vertex $u_{1}$ and that $u_{1} \in \Omega_{2}$. By Theorem 4, the sets

$$
\left\{(x, y): x \in \Omega_{1}, y \in \Omega_{2}\right\} \cup\left\{(w, z): w \in \Delta_{1}, z \in \Delta_{2}\right\}
$$

and

$$
\left\{(w, y): w \in \Delta_{1}, y \in \Omega_{2}\right\} \cup\left\{(x, z): x \in \Omega_{1}, z \in \Delta_{2}\right\}
$$

are the independent neighborhood sets of $G \bullet H_{1}$. Suppose $H_{2}$ is another rooted tree with rooted vertex $u_{2}$ different from $u_{1}$ whose graph is the same with $H_{1}$. Without loss of generality, let $u_{2} \in \Delta_{2}$. We can see that the sets

$$
\left\{(x, y): x \in \Omega_{1}, y \in \Omega_{2}\right\} \cup\left\{(w, z): w \in \Delta_{1}, z \in \Delta_{2}\right\}
$$

and

$$
\left\{(w, y): w \in \Delta_{1}, y \in \Omega_{2}\right\} \cup\left\{(x, z): x \in \Omega_{1}, z \in \Delta_{2}\right\}
$$

are also the independent neighborhood sets of $G \bullet H_{2}$. Thus,

$$
N_{i}\left(G \bullet H_{2}, x\right)=x^{\left|\Omega_{1}\right|\left|\Omega_{2}\right|+\left|\Delta_{1}\right|\left|\Delta_{2}\right|}+x^{\left|\Delta_{1}\right|\left|\Omega_{2}\right|+\left|\Omega_{1}\right|\left|\Delta_{2}\right|} .
$$

Therefore,

$$
N_{i}\left(G \bullet H_{2}, x\right)=N_{i}\left(G \bullet H_{1}, x\right) .
$$


Example 7. Consider the graphs $G$ and $H$ in Figure 5. Let $H_{2}$ be a rooted graph with rooted vertex 1 whose graph is the same with $H$.

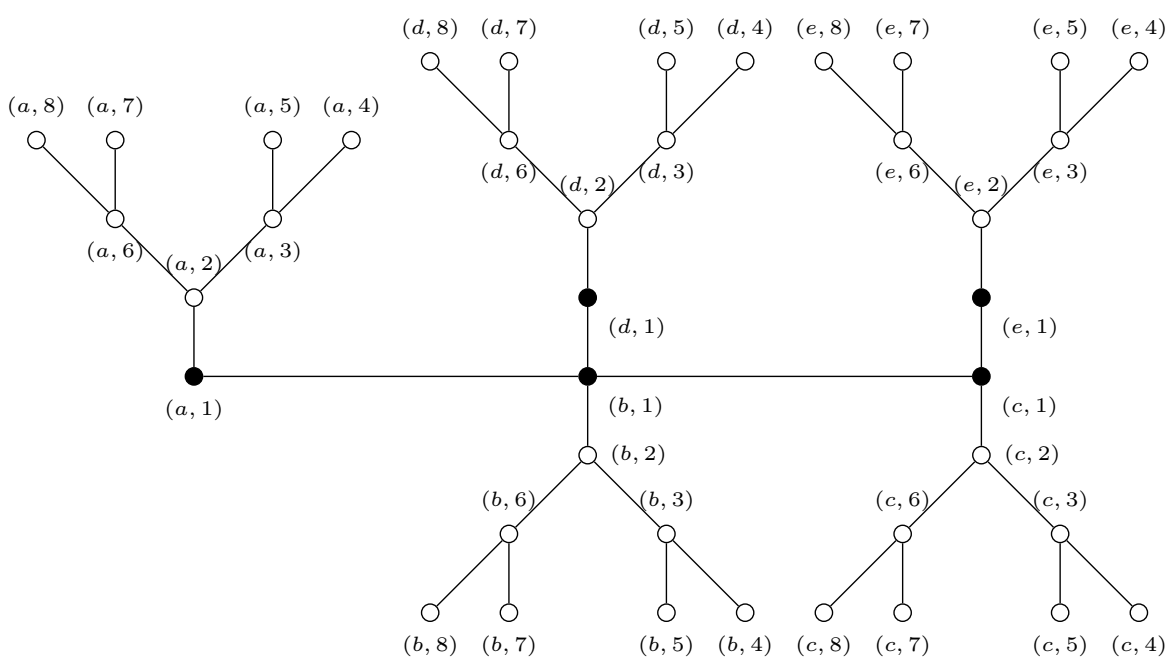

Figure 7: Rooted product of tree $G$ and rooted tree $\mathrm{H}_{2}$

We can verify that the independent neighborhood sets of $G \bullet H_{2}$ are $\{(a, 2),(a, 4)$, $(a, 5),(a, 7),(a, 8),(d, 2),(d, 4),(d, 5),(d, 7),(d, 8),(c, 2),(c, 4),(c, 5),(c, 7),(c, 8),(b, 1)$, $(b, 3),(b, 6),(e, 1),(e, 3),(e, 6)\}$ and $\{(b, 2),(b, 4),(b, 5),(b, 7),(b, 8),(e, 2),(e, 4)$, $(e, 5),(e, 7),(e, 8),(a, 1),(a, 3)(a, 6),(d, 1),(d, 3),(d, 6),(c, 1),(c, 3),(c, 6)\}$. Therefore,

$$
N_{i}\left(G \bullet H_{2}, x\right)=N_{i}\left(G \bullet H_{1}, x\right) .
$$

Theorem 6. Suppose $G$ is the rooted tree with independent neighborhood sets $\Omega_{1}, \Delta_{1}$ and $H$ be any tree with independent neighborhood sets $\Omega_{2}, \Delta_{2}$. Then the independent neighborhood sets of $H \bullet G$ are

$$
\left\{(x, y): x \in \Omega_{2}, y \in \Omega_{1}\right\} \cup\left\{(w, z): w \in \Delta_{2}, z \in \Delta_{1}\right\}
$$

and

$$
\left\{(w, y): w \in \Delta_{2}, y \in \Omega_{1}\right\} \cup\left\{(x, z): x \in \Omega_{2}, z \in \Delta_{1}\right\} .
$$

Proof. The proof can be shown similar to Theorem 4 by just interchanging the first and second coordinates of the ordered pairs of vertices.

Theorem 7. Let $\Omega_{1}, \Delta_{1}$ and $\Omega_{2}, \Delta_{2}$ be the independent neighborhood sets of trees $G$ and $H$, respectively. Then

$$
N_{i}(H \bullet G, x)=N_{i}(G \bullet H, x) .
$$

Proof. Let $G$ and $H$ be trees with independent neighborhood sets $\Omega_{1}, \Delta_{1}$ and $\Omega_{2}, \Delta_{2}$, respectively. Notice that

$$
\left|\left\{\left(x_{1}, y_{1}\right): x_{1} \in \Omega_{1}, y_{1} \in \Omega_{2}\right\}\right|=\left|\left\{\left(x_{2}, y_{2}\right): x_{2} \in \Omega_{2}, y_{2} \in \Omega_{1}\right\}\right|
$$




$$
\begin{aligned}
& \left|\left\{\left(x_{3}, y_{3}\right): x_{3} \in \Delta_{1}, y_{3} \in \Delta_{2}\right\}\right|=\left|\left\{\left(x_{4}, y_{4}\right): x_{4} \in \Delta_{2}, y_{4} \in \Delta_{1}\right\}\right| \\
& \left|\left\{\left(x_{5}, y_{5}\right): x_{5} \in \Delta_{1}, y_{5} \in \Omega_{2}\right\}\right|=\left|\left\{\left(x_{6}, y_{6}\right): x_{5} \in \Omega_{2}, y_{6} \in \Delta_{1}\right\}\right| \\
& \left|\left\{\left(x_{7}, y_{7}\right): x_{7} \in \Omega_{1}, y_{7} \in \Delta_{2}\right\}\right|=\left|\left\{\left(x_{8}, y_{8}\right): x_{8} \in \Delta_{2}, y_{8} \in \Omega_{1}\right\}\right| .
\end{aligned}
$$

Therefore,

$$
N_{i}(H \bullet G, x)=N_{i}(G \bullet H, x)
$$

Example 8. Consider the graphs $G$ and $H$ in Figure 5. Suppose that $G$ is the rooted graph and wihtout loss of generality, let $a$ be its rooted vertex shown in Figure 8.

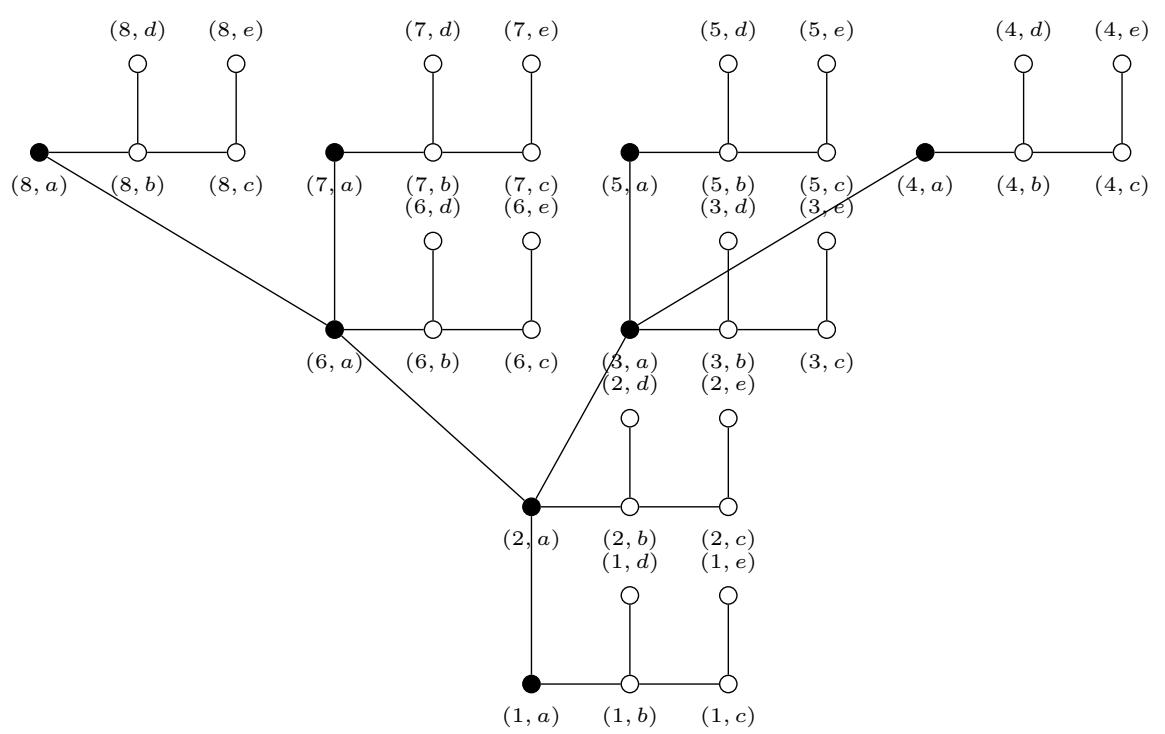

Figure 8: Rooted product of tree $H$ and rooted tree $G$

By Theorem 6 , the independent neighborhood sets of $H \bullet G$ are

$$
\begin{aligned}
\left\{(x, y): x \in \Omega_{2}, y \in \Omega_{1}\right\} \cup\left\{(w, z): w \in \Delta_{2}, z \in \Delta_{1}\right\} \\
=\{(2, a),(2, d),(2, c),(4, a),(4, d),(4, c),(5, a),(5, d),(5, c),(7, a),(7, d),(7, c), \\
\quad(8, a),(8, d),(8, c)\} \cup\{(1, b),(1, e),(3, b),(3, e),(6, b),(6, e)\} \\
=\{(2, a),(2, d),(2, c),(4, a),(4, d),(4, c),(5, a),(5, d),(5, c),(7, a),(7, d),(7, c), \\
\quad(8, a),(8, d),(8, c),(1, b),(1, e),(3, b),(3, e),(6, b),(6, e)\}
\end{aligned}
$$

and

$$
\begin{aligned}
& \left\{(x, y): x \in \Delta_{2}, y \in \Omega_{1}\right\} \cup\left\{(w, z): w \in \Omega_{2}, z \in \Delta_{1}\right\} \\
& =\{(1, a),(1, d),(1, c),(3, a),(3, d),(3, c),(6, a),(6, d),(6, c)\} \cup\{(2, b),(2, e), \\
& \quad(4, b),(4, e),(5, b),(5, e),(7, b),(7, e),(8, b),(8, e)\}
\end{aligned}
$$




$$
\begin{aligned}
=\{ & (1, a),(1, d),(1, c),(3, a),(3, d),(3, c),(6, a),(6, d),(6, c),(2, b),(2, e), \\
& (4, b),(4, e),(5, b),(5, e),(7, b),(7, e),(8, b),(8, e)\} .
\end{aligned}
$$

Furthermore,

$$
\left|\left\{(x, y): x \in \Omega_{2}, y \in \Omega_{1}\right\} \cup\left\{(w, z): w \in \Delta_{2}, z \in \Delta_{1}\right\}\right|=21
$$

and

$$
\left|\left\{(x, y): x \in \Delta_{2}, y \in \Omega_{1}\right\} \cup\left\{(w, z): w \in \Omega_{2}, z \in \Delta_{1}\right\}\right|=19 .
$$

Therefore,

$$
N_{i}(H \bullet G, x)=x^{19}+x^{21}=N_{i}(G \bullet H, x) .
$$

\section{Acknowledgements}

This research is funded by the Department of Science and Technology (DOST), Mindanao State University IIT, Department of Research, Office of the Vice Chancellor for Research and Extension(OVCRE), and Mindanao State University Main Campus (MSU Main).

\section{References}

[1] G.D Birkhoff. A determinant formula for the number of ways of coloring a map. Ann. of Math. (2), 14:42-46, 1912.

[2] F. Buckley and F. Harary. Distance in Graphs. Addison-Wesley Series in Mathematics, 1990.

[3] R. Diestel. Graph Theory. Springer Nature, 2017.

[4] E.J. Farrel. Introduction to matching polynomials. J. Combinatorial Thoery B, 27:75-86, 1979.

[5] P. Zhang G. Chartrand. A First Course in Graph Theory. Dover Publications, Inc, Mineola, New York, 2012.

[6] F. Harary. Graph Theory. Addison-Wesley Publishing Company, 1969.

[7] F. Harary I. Gutman. Generalizations of the matching polynomial. Util. Math, 24:97$106,1983$.

[8] U.S.R. Murthy J.A. Bondy. Graph Theory, Graduate Texts in Mathematics. Springer, 2007.

[9] R.J. Nowakowski J.I. Brown. The neighbourhood polynomial of a graph. Astralasian Journal of Combinatorics, 42:55-68, 2008. 
[10] V. E. Levit and E. Mandrescu. The independence polynomial of a graph-a survey. In Proceedings of the $1^{\text {st }}$ International Conference on Algebraic Informatics, pages 233-254, 2005.

[11] K.B. Murthy and Puttaswamy. On the independent neighbourhood polynomial of graphs. Indian Streams Research Journal, 5(12):1-7, 2015. 